\title{
Measurement of Atmospheric Neutrino Oscillations with the ICECuBe-DeEpCore Detector
}

\author{
Markus Vehring ${ }^{* \dagger}$ \\ III. Physikalisches Institut B, RWTH Aachen University, 52074 Aachen, Germany \\ E-mail: vehring@physik.rwth-aachen.de
}

\begin{abstract}
With its low-energy extension DeEPCoRE, the ICECube Neutrino Observatory, located at the geographic South Pole, measures neutrinos with energies above about $10 \mathrm{GeV}$. With this low energy threshold high statistics of about 150, 000 triggered atmospheric muon neutrinos are recorded per year. This enables the measurement of neutrino oscillations. The oscillation probability depends on the energy and the distance the neutrino traveled to the detector. The oscillation is visible by the energy and zenith dependent disappearance in the recorded muon neutrino rate. Recently, an analysis using three years of data of the completed 86-string detector taken from 2011 to 2014 has achieved a sensitivity approaching that of dedicated oscillation experiments (e.g. MINOS, T2K and Super-Kamiokande-IV). To further improve the sensitivity, this analysis has been extended with data taken from 2010 to 2011 in the 79-string detector configuration, which increases the live time from 953 to 1266 days. The following paper will discuss the analysis method and present results of this muon neutrino disappearance measurement with ICECUBE-DEEPCORE.
\end{abstract}

The European Physical Society Conference on High Energy Physics

22-29 July 2015

Vienna, Austria

* Speaker.

${ }^{\dagger}$ for the IceCube Collaboration (https://icecube.wisc.edu/collaboration/authors) 


\section{Introduction}

In the Standard Model (SM) neutrinos are massless. However, the discovery of atmospheric neutrino oscillations by the Super-Kamiokande Collaboration in 1998 [1] and solar neutrino oscillations by the SNO Collaboration in 2001 [2], requires different mass eigenstates for the different physical neutrinos. Thus, the measurement directly shows physics beyond the SM. Both measurements were awarded the Nobel Prize in Physics in October 2015 [3].

Neutrino oscillations occur due to the flavor eigenstates of neutrinos not being the same as the mass eigenstates [5]. The production and final interaction of neutrinos is mediated via the weak force and is thus coupled to the flavor eigenstates, while the propagation is coupled to the physical particle and thus the mass eigenstates. The probability to measure a certain flavor then depends on the energy and path length of the neutrino.

This analysis focuses on the measurement of the parameters $\sin ^{2}\left(\theta_{23}\right)$ and $\Delta m_{32}^{2}$, which are mostly linked to the oscillations of $v_{\mu}$ into $v_{\tau}$. Since the measurement of this kind of neutrino oscillations is performed by measuring neutrinos produced in the atmosphere, this part of the neutrino oscillations is also called "atmospheric neutrino oscillations" and the parameters are often written as $\sin ^{2}\left(\theta_{\text {atm }}\right)$ and $\Delta m_{\text {atm }}^{2}$.

This analysis is an extension of a previous, published analysis [6].

\section{The ICECUBE Neutrino Observatory}

ICECUBE is a cubic-kilometer sized neutrino detector installed in the glacial ice at the geographic South Pole at depths between $1450 \mathrm{~m}$ and $2450 \mathrm{~m}$ [7]. Detector construction started in 2005 and finished in 2010. It consists of 86 cables (strings) deployed in a hexagonal pattern with 60 optical sensors (DOMs) each. Energy and track reconstruction relies on the optical detection of Cherenkov radiation emitted by secondary particles produced in neutrino interactions in the surrounding ice or the nearby bedrock.

The DEEPCORE sub-array [8] in the lower center of ICECUBE is a region of more densely instrumented strings optimized for low energies. It includes seven central ICECUBE strings and eight special DeEPCore strings. Six of the DEEPCore strings are equipped with DOMs that have a ca. $35 \%$ higher optical efficiency. Fifty DOMs on the DEEPCoRE strings are located in the clearest ice in the bottom part of the detector, whereas ten DOMs form a veto cap above DEEPCORE (see figure 3 for a sketch). The inter-string spacing in DEEPCORE is reduced from $\sim 125 \mathrm{~m}$ to $\sim 65 \mathrm{~m}$ and the inter-DOM spacing is reduced from $17 \mathrm{~m}$ to $7 \mathrm{~m}$ for the DEEPCoRE-strings. This lowers the detection threshold for DEEPCORE to about $10 \mathrm{GeV}$.

This analysis uses four years of data. The first year of data was taken with a not yet completed detector in a 79-string configuration and is in the following called IC79. The data of the following years was taken with a completed 86-string detector and is thus called IC86.

\section{Neutrino Oscillations in ICECUBE-DEEPCORE}

The detection of neutrino oscillations is done by measuring deficit of muon events from muon neutrino charged-current interactions inside ICECUBE compared to the no-oscillation hypothesis. 


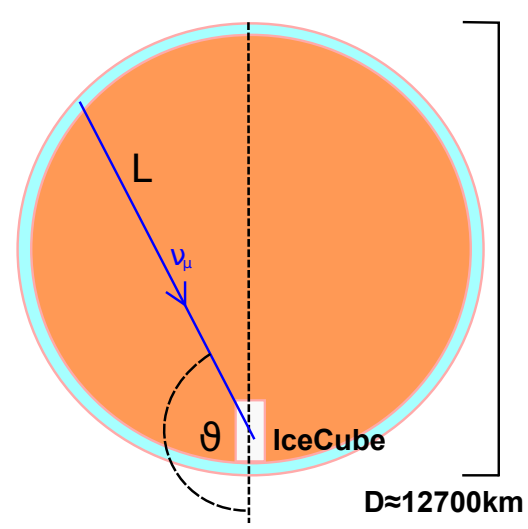

Figure 1: Sketch of the Earth with a $v_{\mu}$ produced in the atmosphere crossing the ICECUBE detector after traversing the Earth.

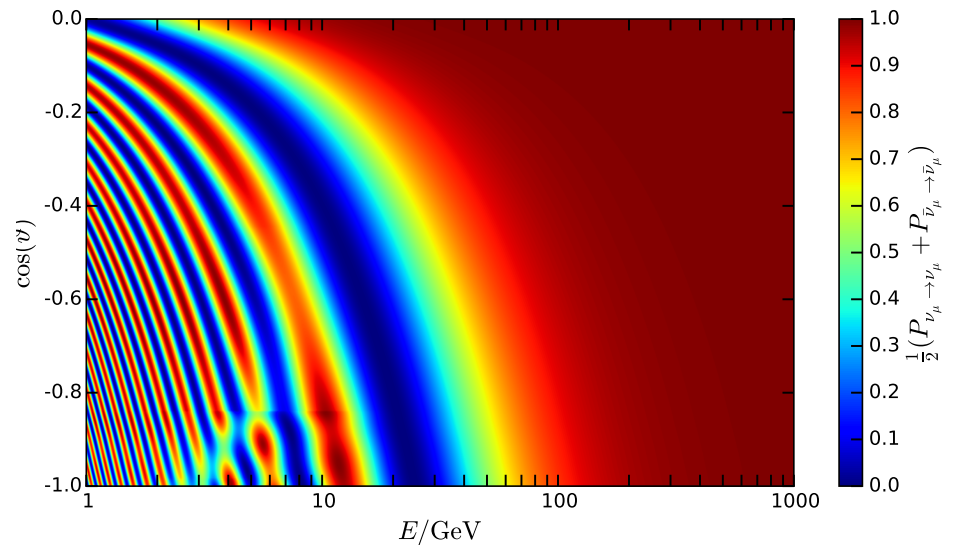

Figure 2: $2 \mathrm{D}$ probability oscillogram in true neutrino energy and cosine of the true neutrino angle for a 1:1 mix of $v_{\mu}$ and $\bar{v}_{\mu}$. (Calculated with NuCraft [10] and neutrino oscillation parameters from [5])

To get the flux for the no-oscillation hypothesis, the known flux of atmospheric neutrinos from Honda et. al. is used [11].

As a good approximation one can neglect the oscillation of muon neutrinos into electron neutrinos and reduce the problem to a two flavor oscillation. In this case, the probability to measure a neutrino produced in its muon flavor as a muon neutrino after a path length $L$ is given by equation 3.1 for the vacuum case.

$$
P\left(v_{\mu} \rightarrow v_{\mu}\right)=1-\sin ^{2}\left(2 \theta_{23}\right) \cdot \sin ^{2}\left(1.27 \cdot \Delta m_{32}^{2} / \mathrm{eV}^{2} \frac{L / \mathrm{km}}{E_{v} / \mathrm{GeV}}\right)
$$

The probability depends on the mixing angle $\theta_{23}$, the squared mass difference $\Delta m_{32}^{2}$ of the neutrino flavors and the energy and path length of the individual neutrino. The energy of the neutrino can be approximated by measuring the track length and recorded light inside the detector. The path length of the neutrino can be obtained from the reconstructed zenith angle of the event (see figure 1). A good approximation is given by equation 3.2.

$$
L \approx D \cdot \cos \left(180^{\circ}-\vartheta\right)
$$

Figure 2 depicts the oscillation probability for different neutrino energies and zenith angles for three flavor oscillations and including matter effects. One can see that the oscillation peaks at about $25 \mathrm{GeV}$ for vertical tracks, well above the $10 \mathrm{GeV}$ threshold of DEEPCoRE, and thus can be measured by ICECUBE-DEEPCORE.

\section{Event Selection}

In a recent analysis of three years of data (IC86) a measurement of the atmospheric neutrino mixing parameters was performed [6]. The following analysis extends this result by another year of data, taken before the detector completion (IC79). Thus the number of strings in DEEPCORE is 


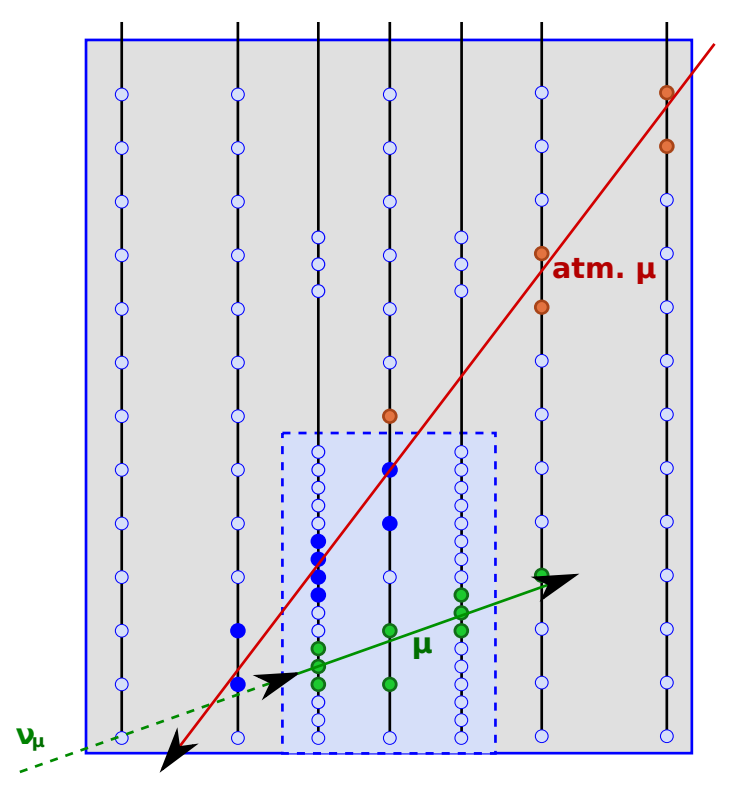

Figure 3: Sketch of the veto algorithm for the first suppression of atmospheric muons. Veto hits inside ICECUBE (red) happen chronologically before the DEEPCORE trigger and thus this event is discarded. For a neutrino that interacts in DEEPCORE, only hits inside DeEPCORE or after the DEEPCORE trigger appear (green).

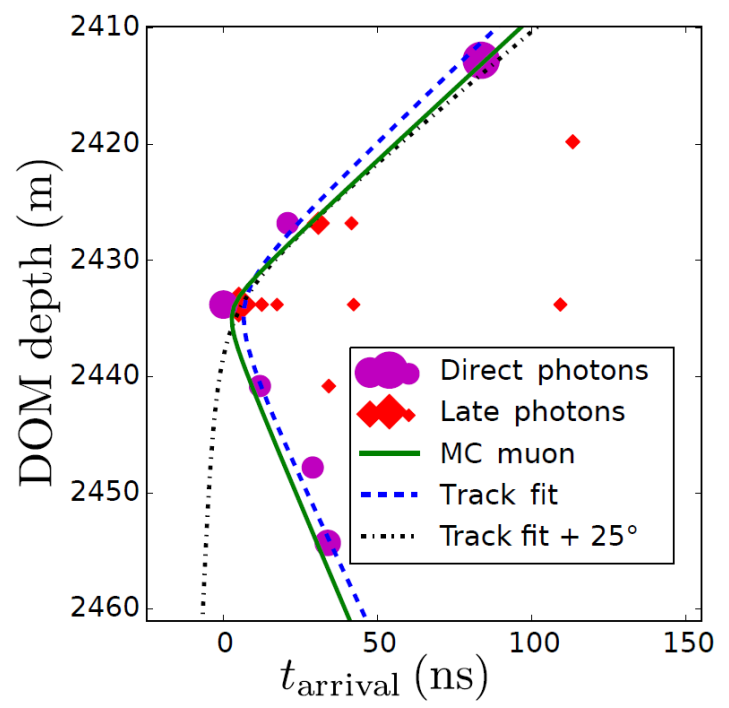

Figure 4: Sketch of the direct-hit selection algorithm (taken from [6]). Direct photons follow a hyperbolic hit-pattern along one string. Scattered photons arrive later and do not follow this pattern.

reduced by two and the overall string count is 79 instead of 86 strings. With a different detector geometry, this extra year of data needs a separate event selection. The IC86 data sample is explained in [9] and [6]. The event selection for the single IC79 year is discussed here.

Events are only used if they trigger the DEEPCORE sub-detector and fulfill the standard DEEPCORE filter requirement [8]. Starting with these events, a first level event selection includes a veto to reduce the amount of atmospheric muons. This is shown in figure 3. An atmospheric muon that enters ICECUBE will produce light in the outer parts of the detector, before it enters the DEEPCORE sub-detector. A neutrino on the other hand can travel undetected through the outer layers of the detector. If the neutrino interacts inside the DEEPCORE fiducial volume, it will produce a muon or a cascade and can trigger DEEPCoRE. Thus, hits that are in the outer layers and happen before the DEEPCORE trigger are expected to be from atmospheric muons. Events that have many of these veto hits or have less than five hits inside DEEPCORE are marked as atmospheric muons. A part of these marked events is processed to the analysis level and is used for a spectrum estimation (template) of the surviving atmospheric muon background.

In the next step events are removed if it is likely that they are dominated by detector noise. Furthermore, events are removed if it is likely that they are from atmospheric muons that travel undetected in corridors between the outer strings and then hit DEEPCORE. If two coincident atmospheric muons produce light in the detector, this can lead to two independent hit clusters. These events are removed, too. At this level the data rate is reduced, so that more sophisticated, computationallyintensive directional reconstructions can be performed. Since atmospheric muons have to have a 

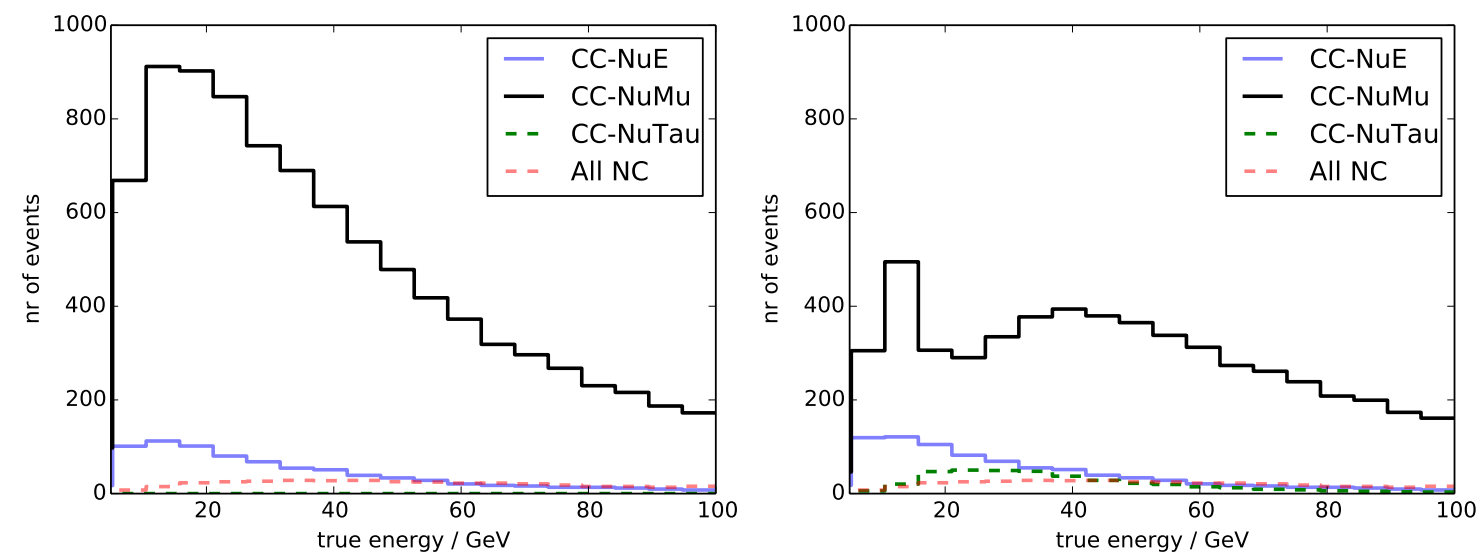

Figure 5: Impact of neutrino oscillations on the true energy spectrum for simulated neutrino events at analysis level for four years of data. In the plot on the left side no neutrino oscillations were taken into account, whereas on the right side three flavor neutrino oscillations were reconsidered (values from [5]). Neutral current interactions are identical between the different flavors and are not separated.

zenith angle below $90^{\circ}$, events that are reconstructed as "down-going" are removed from the sample. As one of the last steps, a machine learning algorithm that utilizes "Boosted Decision Trees" (BDTs) is used to even further separate atmospheric muons from neutrinos. The BDTs utilize different quality parameters from reconstructions. After the BDT-based selection, the data set is dominated by events from neutrino interactions with an estimated purity of around $90 \%$.

Due to the low-energy nature of the bulk of recorded events, only few Cherenkov photons are recorded per event. Some of these photons do not reach the optical sensors on a straight path, but are scattered in the ice and loose their timing information. These scattered photons reduce the quality of reconstructions. For a good reconstruction direct, unscattered photons are needed. Thus, events with only a few of these direct photons are removed in an extra selection step (see figure 4). Direct Cherenkov photons of charged particles produce a hyperbolic hit pattern at the string. If not enough direct hits are found, the event is discarded. Also, events are discarded, if the reduced $\chi^{2}$ for the Cherenkov hypothesis is not good enough. For a more in depth explanation of this see [9].

\section{Data Set Combination and Fit}

At final level there are 6346 events in the combined event sample for four years (IC79: 1172, IC86: 5174). The oscillation effect on the individual simulation contributions is shown in figure 5. Around $50 \%$ of the $v_{\mu}$ events should disappear below $40 \mathrm{GeV}$ and produce the oscillation signal. One can see that a mayor background at this level is due to $v_{\mathrm{e}}$ events. The fit is done in the same way as in [6]. Events of each year are histogrammed in a $8 \times 8$ histogram in cosine of the reconstructed zenith angle form 0 to -1 and the decadic logarithm of the reconstructed energy from $\sim 6.3 \mathrm{GeV}$ to $\sim 56 \mathrm{GeV}$. For every bin the negative logarithm of the poissonian likelihood (nLLH) between data $\left(N_{D}\right)$ and simulation $\left(N_{S}\right)$ is calculated in a simplified way, where constant terms only dependent on $N_{D}$ are omitted.

$$
\mathrm{nLLH}=N_{S}-N_{D} \ln \left(N_{S}\right)
$$


In the end, the nLLH values for the individual years are added together and minimized over the oscillation parameters and nuisance parameters. The mayor difference to [6] lies in the treatment of the individual event samples. Since the detector geometry is different between IC79 and IC86, the events cannot be combined in a trivial way. Both samples have their own set of simulations. In the fit, each year is then treated as a separate entity. The years are linked over oscillation parameters and nuisance parameters. Due to the difference in geometry, the atmospheric muon template, obtained from marked events in the first veto step, is different between IC79 and IC86. Thus the normalization factor for this contribution is not linked. Also, the global normalization for each year is not linked, since the live-time for each year is different. Shared nuisance parameters are the scattering length in the refrozen hole-ice around the DOMs, the optical efficiency of the DOMs, the $v_{\mu}$ normalization, the $v_{\mathrm{e}}$ deviation from the $v_{\mu}$ normalization, the primary cosmic ray index and the value of $\theta_{13}$.

\section{Result}

Figure 6 shows the $L / E$-projection of experimental data and simulation. The blue line shows the Monte-Carlo simulation for the best fit oscillation and nuisance parameters, whereas the reddashed line shows the case without oscillations. The data clearly favors the oscillation case and matches the best fit simulation well. Events with an $L / E$ lower than $100 \frac{\mathrm{km}}{\mathrm{GeV}}$ do not show strong oscillation effects and are used for normalization.

The result of a full parameter scan can be seen in figure 7. The $90 \%$ C.L. contour shrinks in regard to the three year analysis (IC2014) and the best fit parameters shift to a higher mass splitting and a larger mixing angle. The values the best fit are:

$$
\sin ^{2}\left(\theta_{23}\right)=0.54_{-0.13}^{+0.08} \quad \text { and } \quad \Delta m_{32}^{2}=2.80_{-0.16}^{+0.20} \cdot 10^{-3} \mathrm{eV}^{2}
$$

This is an improvement over the previous result of $\sin ^{2}\left(\theta_{23}\right)=0.53_{-0.12}^{+0.09}$ and $\Delta m_{32}^{2}=2.72_{-0.19}^{+0.20}$. $10^{-3} \mathrm{eV}^{2}$. The improvement is limited by systematic errors, which are more pronounced for the IC79 sample, as IC79 has a lower string density in DEEPCORE.

To evaluate the fit stability, a fit was also performed for the two flavor scheme, not limited to physical $\sin ^{2}\left(2 \theta_{23}\right)$ values, which gave consistent results.

\section{Conclusion and Outlook}

The presented extension of the three-year result of the ICECUBE muon-neutrino disappearance analysis yields an increased sensitivity, especially for the mass splitting. Since the detector is completed an existing event selection can be used for every new year of data and more years can now easily be added to the existing data.

New reconstructions that do not need the direct photon requirement are being developed and thus will raise the statistics by a factor of ten for the same live-time, as well as provide better reconstruction performance. Higher statistics and better reconstructions with a handle on particle identification will give the opportunity to use a third dimension in the fit. All these advancements should greatly impact the precision of the measurement with the ICECUBE. 

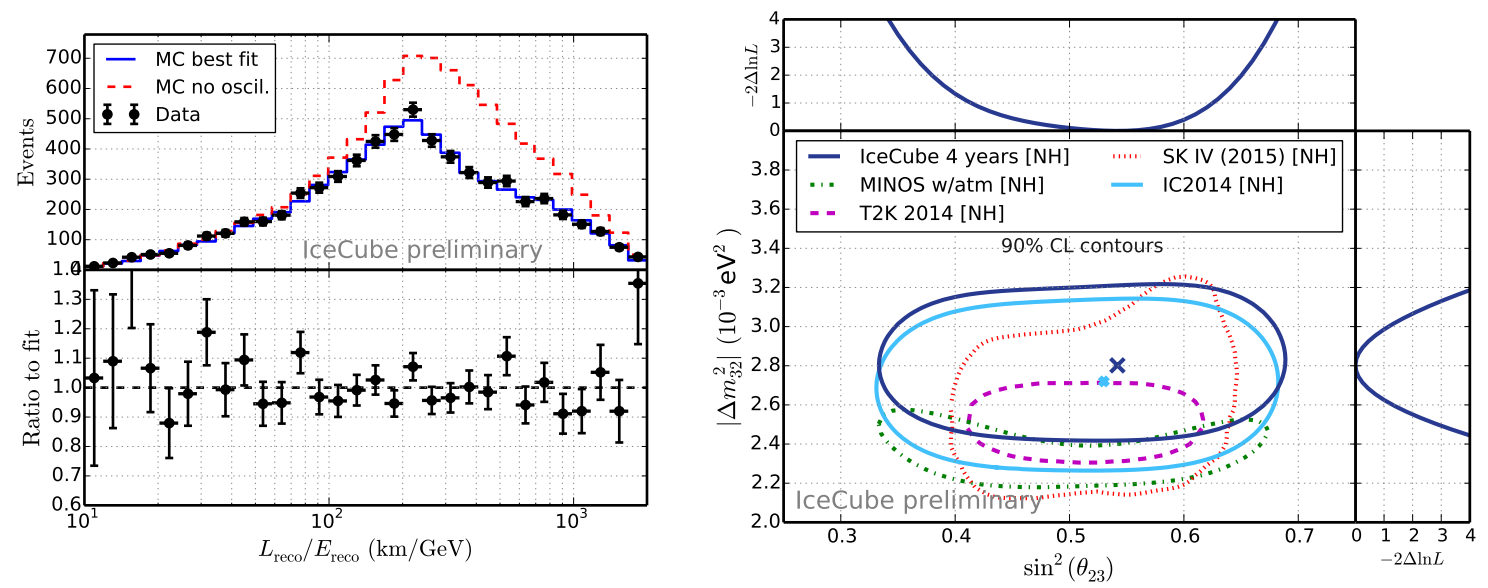

Figure 6: L/E projection for data (black dots), Figure 7: Parameter scan in $\sin ^{2}\left(\theta_{23}\right)-\Delta m_{32}^{2}$-plane and simulation with the best fit oscillation parameters (blue solid line) and simulation for the nooscillation hypothesis (red dashed line) for four years of data. The nuisance parameters for both simulation curves are identical. comparison to other measurements. The best fit is at $\sin ^{2}\left(\theta_{23}\right)=0.54_{-0.13}^{+0.08}$ and $\Delta m_{32}^{2}=2.80_{-0.16}^{+0.20} \cdot 10^{-3} \mathrm{eV}^{2}$. With contours taken from [6][12][13][14].

\section{References}

[1] Y. Fukuda et al. [Super-Kamiokande Collaboration], Phys. Rev. Lett. 81:1562-1567 (1998) [arXiv:hep-ex/9807003].

[2] B. Aharmim et al. [SNO Collaboration], Phys. Rev. Lett. 87, 071301 (2001) [arXiv:nucl-ex/0106015].

[3] Royal Swedish Academy of Sciences, www.nobelprize.org

[4] B. Pontecorvo, Sov. Phys. JETP 33:549-551 (1957).

[5] K.A. Olive et al. [Particle Data Group], Chin. Phys. C38, 090001 (2014).

[6] M.G. Aartsen et al. [ICECubE Collaboration], Phys. Rev. D91, 072004 (2015) [arXiv:1410.7227].

[7] A. Achterberg et al. [ICECuBE Collaboration], Astropart. Phys. 26:155-173 (2006) [arXiv:1109.6096].

[8] R. Abbasi et al. [IcECuBE Collaboration], Astropart. Phys. 35:615-624, (2012) [arXiv:1109.6096].

[9] J. P. Yáñez Garza, Ph.D. thesis, Humboldt-Universität zu Berlin (2014).

[10] M. Wallraff and C. Wiebusch, Comput. Phys. Commun. (forthcoming) [arXiv:1409.1387].

[11] M. Honda, T. Kajita, K. Kasahara, S. Midorikawa and T. Sanuki, Phys. Rev. D75, 043006 (2007) [arXiv:astro-ph/0611418].

[12] Roger Wendell (for the Super-Kamiokande Collaboration), AIP Conf. Proc. 1666, 100001 (2015) arXiv: 1412.5234.

[13] P. Adamson et al. [MINOS Collaboration], Phys. Rev. Lett. 112, 191801 (2014) [arXiv:1403.0867].

[14] K. Abe et al. [T2K Collaboration], Phys. Rev. D91, 072010 (2015) [arXiv:1502.01550]. 